\title{
NUEVOS EXCLUIDOS EN EL SISTEMA EDUCACIONAL CHILENO: PROBLEMAS Y DESAFÍOS
}

\section{New Excluded in the Chilean Educational System: Problems and Challenges}

\author{
JUAN CORNEJO ESPEJO*1 \\ http://orcid.org/0000-0002-6436-0908 \\ Recibido: 27-02- 2017 \\ Revisado: 04-04-2017 \\ *Universidad Católica del Maule. Chile. \\ Aceptado: 20-11-2017
}

Correspondencia: juancornejoespejo@gmail.com

Resumen: El artículo es una reflexión crítica acerca de los alcances y desafíos de la inclusión educativa, ya que esta se ha centrado casi exclusivamente en la atención de estudiantes con necesidades educativas especiales. Los otros grupos excluidos en la escuela tradicionalmente han sido omitidos, invisibilizados o silenciados, motivo por el cual su irrupción en escena supone nuevos retos. El artículo está dividido en tres partes: la primera problematiza los significados e implicancias de la inclusión educativa. La segunda cuestiona los proyectos integradores que promueven la asimilación, normalización e invisibilización de las diferencias. En la tercera parte se esbozan algunas ideas que apuestan por la deconstrucción de los paradigmas que sustentan la exclusión en tanto exigencia para una auténtica inclusión educativa.

Palabras clave: inclusión educativa; exclusión; sistema educacional; desafíos.

\begin{abstract}
The article is a critical reflection on the scope and challenges of educational inclusion, since the subject has been focused almost exclusively on the attention of students with special educational needs. The other excluded groups in the school traditionally have been omitted, invisibilized or silenced, reason why its irruption in scene supposes new challenges. The article is divided into three parts: The first problematizes the meanings and implications of educational inclusion. The second questions integration projects that promote the assimilation, normalization and invisibility of differences. The third part sketches some ideas that bet on the deconstruction of the paradigms that support the exclusion as a requirement for an authentic educational inclusion.
\end{abstract}

Keywords: educational inclusion; exclusion; educational system; challenges.

\footnotetext{
${ }^{1}$ Doctor en Ciencia Política por el Instituto Universitario de Pesquisas de Rio de Janeiro (IUPERJ). Doctor en Estudios Americanos por la Universidad de Santiago de Chile (USACH). Magíster en Educación por USACH. Magíster en Historia por USACH. Magíster en Ciencia Política por la Pontificia Universidad Católica de Chile. Magíster en Ciencias Sociales por el Instituto Latinoamericano de Doctrina y Estudios Sociales. Becario Posdoctoral por The Manchester University - Center of Sexuality and Culture- CONICYT 74150025. Académico del Departamento de Fundamentos de la Educación de la Universidad Católica del Maule. Líneas de Investigación: disidencias sexuales; género y educación; inclusión educativa.
} 


\section{INTRODUCCIÓN1}

Tradicionalmente, la formación de profesores en Chile ha tendido a "invisibilizar" las diferencias de los estudiantes con los que deberán trabajar los futuros pedagogos. Y aun cuando ello no es exclusivo del sistema escolar chileno, tal condición se ha hecho más notoria en razón de lo persistente de las políticas "asimilacionistas" que han intentado subsanar esa invisibilización, por medio de la integración de los grupos minoritarios o disidentes a la cultura y valores hegemónicos. En esta línea el filósofo Martín Hopenhayn (2002) sostiene que los conflictos del multiculturalismo se vinculan históricamente a la "dialéctica de la negación del otro".

Podría argumentarse que la negación del otro es negación del multiculturalismo, es decir, el reconocimiento unilateral de una cultura como válida frente a otras que se les niega legitimidad. Pero también es una forma opresiva de asumir el multiculturalismo: se reconoce la presencia de otras identidades, pero solo para degradarlas ontológicamente..... Otra forma de negación del otro fue la aculturación de los pueblos indígenas y afrolatinoamericanos, vale decir, la negación de su propio universo simbólico con el fin de disciplinarlos en el trabajo productivo, la ideología del Estado-Nación, el espíritu racionalista y el uso de una lengua europea.... La negación del otro adquirió luego el rostro más visible de la exclusión social y aún lo perpetúa.... En efecto, un rasgo cotidiano de exclusión ha sido la recurrente invisibilización de la diferencia.

Con todo, no se puede desconocer que ya desde fines de los noventa ha habido una preocupación creciente de parte de las autoridades por sensibilizar a los futuros profesores de las distintas disciplinas acerca de la necesidad de "integrar" a los estudiantes con necesidades educativas especiales (NEE) a los proyectos de formación de los estudiantes considerados "normales"2. Sin embargo, ese esfuerzo, pese a las fuertes inversiones, se ha visto limitado no sólo por la falta de preparación de los profesores para lidiar con este tipo de estudiantes, sino también por la ausencia

\footnotetext{
${ }^{1}$ Proyecto de investigación financiado por el Plan de Mejoramiento UCM 1310 de formación inicial de profesores.

2 Desde el punto de vista legal es posible visualizar un conjunto de normativas, leyes y decretos que buscan asegurar una educación de calidad para los estudiantes que presentan NEE, ya sean de carácter permanente o transitorio. Entre las más recientes destacan: Decreto № 1/98 y N1300/2002, que aprueba planes y programas de estudio para alumnos con trastornos específicos del lenguaje. Ley 19.284/1994, que establece normas para la plena integración social de las personas con discapacidad. Decreto $N^{\circ} 201 / 2008$, que ratifica la Convención Internacional sobre los Derechos de las Personas con discapacidad. Ley 20.201/2007, y su reglamento, Decreto N $170 / 2009$, Orientaciones técnico-pedagógica para la evaluación integral. Art. 34 del DFL № 2/2009, que mandata al MINEDUC para establecer criterios y orientaciones de adecuación curricular. Ley 20.422/2010, de igualdad de oportunidades e inclusión social para las Personas con discapacidad. Art. 3, señala entre sus principios el Diseño Universal de Aprendizaje (DUA), Decreto $N^{\circ} 83 / 2015$ que incluye orientaciones para implementar adecuaciones curriculares para personas con necesidades educativas. Ley $20.845 / 2015$, de Inclusión Escolar que regula la admisión de los estudiantes, elimina el financiamiento compartido y prohíbe el lucro en establecimientos educacionales que reciben aportes del Estado. Pese a los avances, en muchos aspectos pioneros en Latinoamérica, se verifica que la inclusión educativa en Chile ha estado focalizada fundamentalmente en la atención de estudiantes descendidos con NEE.
} 
de proyectos educativos efectivamente inclusivos. En el mejor de los casos han sido proyectos de integración ${ }^{3}$ que buscan poner en contacto a estudiantes con NEE con el resto de la comunidad educativa, a través de la puesta en común de ciertos espacios al interior de la escuela o bien por el apoyo de ciertos profesionales que refuerzan la labor pedagógica. Y si bien en 2015 se promulgó la Ley 20.845 de Inclusión Escolar (Biblioteca del Congreso Nacional de Chile, 2015), sus alcances son muy limitados, pues solo hace referencia a la obligación que tienen los establecimientos educacionales que reciben aportes financieros del Estado de admitir a todos los estudiantes, eliminando tanto las pruebas de selección académica como cualquier otro impedimento que justifique cualquier forma de exclusión.

Con todo, nada se dice respecto de las distintas formas de exclusión o discriminación presentes en los sistemas escolares formales, ni tampoco de la obligatoriedad que debiese pesar también sobre los establecimientos que no reciben aportes económicos del Estado, en el sentido de respetar el derecho a la educación que tienen aquellos estudiantes menos aventajados académicamente, con capacidades diferentes, pertenecientes a algún grupo minoritario o considerados disidentes de un cierto orden. Respecto de las otras diferencias presentes en la escuela motivadas por la pertenencia a grupos étnicos minoritarios, pueblos originarios, por la condición de migrante, portador de alguna deficiencia física (capacidades diferentes), por una orientación sexual o identidades genéricas disidentes del ideal heteronormativo, entre otras, no sólo son descuidadas, sino que las políticas hasta ahora implementadas que buscan garantizar un acceso equitativo e igualdad de oportunidades entre todos los estudiantes son aún insuficientes. Este diagnóstico no solo está presente en reflexiones de especialistas (Blanco, 2011) sino en las propias discusiones de los distintos equipos de trabajo sobre la materia del Ministerio de Educación de Chile (2016).

\footnotetext{
3 Desde fines de los ochenta, por exhortaciones de organismo internacionales, se comienza a promover la "integración" de los denominados niños "discapacitados" en la educación común superando la opción de una educación segregada. Este movimiento integracionista se funda en la idea de la "normalización" como principio rector en el ámbito de los servicios asistenciales a personas discapacitadas (Van Steenlandt, 1991). No obstante lo anterior, quedaron al margen las minorías étnicas y los minusválidos. Y fueron, precisamente, los grupos de presión encabezados por las Asociaciones de Padres de niños minusválidos en Estados Unidos quienes actuaron de forma decisiva sobre los derechos civiles de los discapacitados. Estos grupos de presión se replantearon el derecho de sus hijos a la escolarización plena, de manera que pudieran compartir experiencias con otros niños, oponiéndose a la estigmatización que representa el etiquetaje de categorías para emplazarlos en un sistema educativo paralelo y segregado que no les permitiera compartir el medio en que se desenvuelve la mayoría (Lavilla, 2013). Pese a los avances que supuso la idea de integración, ella no consideró a otros grupos excluidos y/o discriminados en el espacio escolar (indígenas, minorías étnicas, migrantes, disidentes sexuales, entre otros), ni tampoco implicó una noción amplia de inclusión que entendiese a la propia comunidad escolar como una comunidad de diferencias donde todos se reconocen en sus diferencias. Con todo, se ha reconocer que la integración, más allá de sus limitaciones, fue y continúa siendo una apuesta por el quiebre de los modelos asimilacionistas, normalizadores u homogeneizantes, cuyo impacto se deja sentir no sólo en plano de los derechos a un acceso universal a la educación sino también en los propios aprendizajes que se ven restringidos en sus posibilidades de alcanzar los niveles óptimos de acuerdo a las capacidades de cada estudiante. Dicho en otros términos, en un primer momento hubo una demanda por una escuela que "integrara" a los niños "distintos" y, posteriormente, que la escuela fuera "diferente" para que pudiera "incluirlos a todos" (Blanco, 1992; Devalle de Rendo y Vega, 2009; Casanova, 2011).
} 
Probablemente, los avances más significativos en esta línea, en un intento por prevenir la violencia y exclusiones que esta genera, se han canalizado a través de la denominada ley antibullying o Ley 20.536 Sobre Violencia Escolar (Biblioteca del Congreso Nacional de Chile, 2011). Esta ley se complementó con la Ley 20.609, denominada ley Zamudio o antidiscriminación (Biblioteca del Congreso Nacional de Chile, 2012), cuyo propósito es sancionar cualquier discriminación arbitraria fundada en la raza, nacionalidad, situación socioeconómica, idioma, ideología, opinión política, religiosa, creencia, sexo, orientación sexual, identidad de género, estado civil, edad, filiación, apariencia personal, enfermedad o discapacidad.

Es más, ni aún dentro del ámbito de las NEE los esfuerzos inclusivos han cubierto a todos los estudiantes. No es un secreto que, si bien hay un interés creciente por los estudiantes descendidos o con menor rendimiento, esa preocupación se desvanece tratándose de los estudiantes talentosos. Estudiantes que, además de no contar con programas educativos que potencien sus capacidades, normalmente son objeto de hostigamiento de parte de sus compañeros o descuido de parte de sus profesores ${ }^{4}$.

En ese escenario, la inclusión educativa se torna aún más compleja, no solo por la invisibilización, falta de atención y exclusión social de esos grupos, sino agravada debido a la segmentación social que caracteriza a la sociedad chilena y que se reproduce en la escuela (Programa de las Naciones Unidas para el Desarrollo, 2017; Organización de las Naciones Unidas para la Educación, la Ciencia y la Cultura, 2011; García Huidobro, 2007). Estos antecedentes dejan de manifiesto que la formación de profesores es aún deficitaria en la mayor parte de las universidades del país, en lo que respecta al reconocimiento de los grupos o personas discriminadas, u objeto de exclusión en la escuela, atención a la diversidad e inclusión de las diferencias; pues todavía no hay plena conciencia de que la escuela es fundamentalmente "una comunidad de diferencias" (Rhoads, 1994). Deficiencias que además se expresan en la falta de competencias, habilidades y estrategias pedagógicas para el trabajo con grupos excluidos desde una perspectiva de una pedagogía de la diversidad y la inclusión (Aránzazu y Aguado, 2002; Aguado, 2009).

En las siguientes páginas se intenta reflexionar acerca de los problemas y desafíos que supone para las instituciones responsables de formación de profesores el tema de la inclusión educativa, especialmente en un momento en que el abanico de

\footnotetext{
${ }^{4}$ La excepción la constituye el PENTA - UC de la Universidad Católica de Chile. A esa iniciativa se han sumado otros programas de similares características en universidades regionales (DELTA UCN - Universidad Católica del Norte; BETA PUCV - Universidad Católica de Valparaíso; Semilla - Universidad Católica del Maule; Talento UdeCUniversidad de Concepción; PROENTA UFRO-Universidad de la Frontera; ALTA UACh-Universidad Austral de Chile). No obstante, su alcance es aún limitado considerando el número de estudiantes que asisten a estos programas y a que no alcanzan a cubrir todo el país.
} 
excluidos de los sistemas escolares formales pareciera haberse ampliado 0 redescubierto su existencia, en razón de que las diferencias en la escuela históricamente han sido negadas, omitidas, invisibilizadas 0 silenciadas, promoviéndose más bien la homogeneización, normalización o sanción de las disidencias (Núñez, 2011). No se pueden obviar tampoco las discordancias e incongruencias en la puesta en práctica de las normativas y políticas del Ministerio de Educación (MINEDUC); si bien se reconocen avances en materia de inclusión en el plano normativo, la implementación de esas políticas es aún deficitaria.

En lo que respecta a la estructura del artículo, la primera parte problematiza los significados e implicancias de la inclusión educativa, especialmente en la formación de profesores, pero también en lo referente a sus tendencias investigativas y puestas en práctica que limitan o distorsionan muchas veces su finalidad última, que no es otra que una escuela sin exclusiones. En la segunda parte se cuestionan los proyectos integradores en educación presentes en políticas parcializadas, que piensan en unos pero omiten a los otros; es decir, políticas colonizadoras, asimilacionistas (Cornejo, 2012a), invisibilizadoras de las diferencias, normalizadoras o que no cuestionan los sustratos ideológicos que preservan un cierto orden fundado en el colonialismo (Maldonado, 2007), la xenofobia, la jerarquía de las sexualidades, el machismo, heterosexismo y la homofobia (Borrillo, 2001, 2009), entre otros. En la tercera parte y final se esbozan algunas ideas que apuestan por la deconstrucción de los componentes ideológicos que sustentan la exclusión, pues una auténtica inclusión educativa no es aquella que se conforma con la integración al espacio escolar de los históricamente marginados o intenta resolver los problemas derivados de esa convivencia, sino aquella educación que ambiciona un nuevo orden basado en la equidad, pero no apenas en el plano jurídico, de lo políticamente correcto o restringido a las desigualdades socioeconómicas (Sawaia, 2002), sino que tiene en consideración las subjetividades, deseos y voces de los sujetos objeto de la inclusión, al tiempo que promueve un orden donde la diferencia es un valor.

\section{PRIMERA PARTE}

\section{Contexto de la discusión}

Desde la Declaración Mundial de la Educación para todos (1990) la inclusión educativa, especialmente de grupos vulnerables a la discriminación y exclusión social se ha convertido en una condición y requisito ineludible de todo sistema educacional, tanto desde el punto de vista de las exigencias que supone la inclusión de nuevos estudiantes, como de la formación de profesores capaces de dar respuesta a los nuevos requerimientos de un mundo complejo y diverso.

Es decir, la inclusión se ha convertido no solo en una pieza clave de los planes de acción propuestos por todos los documentos posteriores de la Organización de las 
Naciones Unidas para la Educación, la Ciencia y la Cultura (UNESCO), sino en una premisa ética. La Declaración de Salamanca (1994), La educación inclusiva. El camino hacia el futuro (2008), las Directrices sobre políticas de inclusión en la educación (2009) y la Declaración de Incheon-Educación 2030 (2015) reiteradamente han insistido sobre la urgencia del tema a través de la incorporación de nuevas temáticas.

En el escenario mundial actual, caracterizado por las constantes transformaciones derivadas del proceso globalizador e internacionalización de las economías, cada vez es más necesario que las universidades replanteen sus objetivos y funciones (Didriksson, 2000; Irigoyen, Jiménez y Acuña, 2011). Esta nueva configuración supone redefinir los perfiles de ingreso y egreso, la gestión curricular, los modelos de carreras, su organización, la preparación de los académicos y la manera de relacionarse con las otras instituciones, entre otras exigencias. Es decir, las políticas educativas en el ámbito universitario han de orientarse a formar profesionales para los nuevos tiempos, lo que implica pensar, trabajar y tomar decisiones en colaboración con otros profesionales (Orozco, 2000). No menos importante es la incorporación de los saberes humanos, sociales, éticos y filosóficos a fin de formar personas en vista de su progreso individual y social. En lo que a inclusión educativa se refiere, es urgente pasar de una noción restringida de integración, a una más amplia que considere no solo a los niños y jóvenes con NEE, sino que incluya a otros grupos tradicionalmente marginados, invisibilizados o excluidos de los sistemas escolares formales.

Se puede concebir el concepto más amplio de educación inclusiva como un principio rector general para reforzar la educación para el desarrollo sostenible, el aprendizaje a lo largo de toda la vida para todos y un acceso a las oportunidades de aprendizaje en condiciones de igualdad para todos los niveles de la sociedad (Organización de las Naciones Unidas para la Educación, la Ciencia y la Cultura, 2008, p. 3).

La riqueza de significado de la educación inclusiva requiere, además, la incorporación de distintos saberes y enfoques de análisis, a fin de articular la multiplicidad de variables: personales, sociales, políticas y escolares (Pedró, 2012). Es decir, es necesario adoptar un enfoque holístico, una perspectiva ecológico-sistémica 0 "ecología de la equidad" de acuerdo a la denominación de Ainscow, Dyson, Goldrick, y West (2012), que mediatice las tensiones que se ejercen sobre la escuela, tanto desde el exterior como del interior (Echieta, 2013), con el propósito de optimizar las competencias que han de desarrollar los profesionales de la educación para hacer frente a la diversidad del estudiantado. No menos importante es la valoración y reconocimiento de todos los estudiantes, de modo que todos se sientan incluidos y considerados individual y colectivamente.

Entendemos que el reconocimiento es la condición básica para vencer la fragilidad humana y lograr la construcción como sujeto. Consiste en la aceptación de los demás de la singularidad y necesidades de cada 
individuo, junto con la invitación a que participe en las diversas formas de relación social. El reconocimiento como aceptación de las singularidades (Puig, 2012, p. 102).

El reconocimiento de los otros como iguales o "igualdad de reconocimiento" se ha constituido hoy en una condición básica al punto que:

El reconocimiento de la dignidad de personas o grupos constituye el elemento esencial de nuestro concepto de justicia.... Hay formas de trato socialmente injustas en las que lo que está en juego no es la distribución de bienes o derechos, sino la ausencia de afectos y cuidado o de estima social, que hurtan la dignidad o el honor (Honneth, 2010, pp. 14-15).

En este sentido las universidades son espacios privilegiados para el desarrollo de capacidades que permitan aprendizajes significativos desde el punto de vista deontológico, de la formación ciudadana y formación humana de los futuros profesores en tanto sujetos miembros de una sociedad democrática e inclusiva.

\section{Tensiones en materia de inclusión educativa}

No se puede obviar el carácter polisémico con que se ha revestido la noción de inclusión educativa. Pese a sus múltiples significados, la mayor parte de los estudiosos en el tema coinciden en que ella se refiere a la

transformación de las culturas de las escuelas para aumentar el acceso o presencia de todos los estudiantes (no solo de los grupos marginados o vulnerables), incrementar la aceptación de todo el alumnado, maximizar su participación en múltiples ámbitos de actividad, y aumentar su rendimiento (Artiles, Kozleski, Dorn, y Christensen, 2006, p. 67).

No obstante, esa conceptualización que amplía a los posibles beneficiarios de las políticas o acciones inclusivas, no restringiéndolo a los estudiantes vulnerables 0 pertenecientes a grupos minoritarios, no ha resultado una discusión fácil o libre de tensiones (Artiles y Kozleski, 2014).

En la práctica se verifican algunas tendencias que de alguna forma distorsionan el intento de ampliar su compresión. La primera de ellas, quizás la más recurrente, es que los estudios referidos al tema se han tendido a concentrar en los estudiantes con NEE descendidos o con problemas de aprendizaje. Los estudiantes talentosos que son una forma inversa de NEE, como ya se señaló, normalmente han sido descuidados.

Otra corriente no menos importante se ha focalizado en el aula o la escuela, descuidando las intersecciones de las otras identidades de los estudiantes (clase social, lengua, género, pertenencia étnica, etc.). Una tercera tendencia ha entendido la inclusión como el lugar donde los estudiantes con NEE reciben una educación 
particular en el mismo entorno del aula ordinaria, especialmente a través del apoyo que prestan otros profesionales.

Una cuarta tendencia focalizada en el establecimiento educacional, pese a haber hecho propuestas o adoptado medidas en vista de optimizar los procesos inclusivos, ha tendido a no documentar sus resultados o los alcances de las medidas propuestas, desconociéndose su efectividad (Artiles y Kozleski, 2014).

Un elemento no menos significativo que explica algunas de las dificultades de enraizamiento de la inclusión educativa en el ámbito escolar en los países del tercer mundo, es el hecho de que el modelo se originó en los países del primer mundo, esparciéndose luego al resto a través de lo que se denominó una "agenda global". Esta situación, de alguna forma, ha dificultado tanto su compresión como la necesidad de su implementación, en tanto exigencia de una escuela que busca atender sin restricciones a todos los que acceden a ella.

En 1990, la Declaración Mundial de la Educación para todos de la UNESCO (Organización de las Naciones Unidas para la Educación, la Ciencia y la Cultura, 1990), con el propósito de buscar la universalización de la educación, reconoció la necesidad de suprimir la disparidad educativa, particularmente en grupos vulnerables a la discriminación y la exclusión. A partir de esta declaración se ha manejado el concepto de Educación para Todos (EFA Education for All) tanto desde UNESCO como de otras agencias de cooperación internacional, a fin de promover un mundo en el que todos los niños y niñas tengan acceso y se les garantice que recibirán una educación de calidad.

Otro hito importante fue la Declaración de Salamanca, basada en el principio de que todos los estudiantes que tuviesen algún requerimiento educativo especial debían ser asistidos y atendidos por los respectivos sistemas educacionales nacionales.

Actualmente existe consenso en que la inclusión educativa está comprometida con una agenda de justicia y equidad, enmarcada en el contexto de los derechos individuales. Con todo, en la práctica se constata que, tanto la producción intelectual en torno al tema como la formación de profesores, distan de los objetivos originales.

En este sentido, no se puede desconocer el desafío que supone compatibilizar programas de formación de profesores para la diversidad con programas generalistas (Arroyo, 2008). Lo cierto es que no habría problema en ello si esa generalización garantizase el acceso y la permanencia exitosa de todos en el sistema escolar, por medio de prácticas curriculares diversificadas y ajustadas a los diferentes ritmos e intereses de aprendizaje (Santos, 2013).

Sin embargo, lo que suele ocurrir es la organización de un currículo que capacita a los profesores para hacer clases en cualquier escuela y a distintos tipos de estudiantes, sin consideración a sus diferencias y peculiaridades, sometiéndolos a todos a un proyecto único de docencia y de formación. De este modo, cuando se concibe el currículo como único y obligatorio, la diferencia queda condicionada no a una perspectiva universal en el sentido de ser derecho de todos, sino a la 
homogeneización de culturas y prácticas, como si "todos" representase una única forma de ser, y no a la diversidad.

Por otra parte, las concepciones generalistas presentes en la mayor parte de los sistemas escolares latinoamericanos y en la formación de sus profesores, evidencian un carácter monolítico, reproduciendo y reforzando patrones que convierten las diferencias en desigualdades.

Comprender tales contradicciones torna posible una relación cooperativa que viabiliza una propuesta de mediación intercultural inclusiva desde una perspectiva decolonial (Maldonado, 2007). La perspectiva intercultural, por su parte, presupone asumir las diferencias como condición para avanzar en concepciones y prácticas de educación, docencia y de formación de profesores que favorezcan la comprensión de tales procesos en diferentes contextos históricos y nacionales, repensando y deconstruyendo los patrones clasificatorios y normalizadores presentes en el universo escolar (Poblete, 2009).

Asimismo, el enfoque intercultural se proyecta como una mirada hacia la diversidad de los estudiantes, familias y comunidades que reconocen dicha diversidad y asumen un compromiso por la igualdad de oportunidades y una escuela buena para todos (Aguado, 2009). La diferencia se impone, así, como un tema central en la formación de profesores, considerando que el núcleo estructurante del sistema educacional está normado por un patrón único que se resiste a desprenderse de las jerarquías sociales, raciales, de género, sexuales, territoriales, generacionales, etc. En otras palabras, la formación de profesores para la diversidad exige un diálogo que favorezca la redefinición de concepciones de formación, de docencia, de conocimientos, de enseñanza y aprendizaje a partir de la reinvención de la realidad que los colectivos diversos traen y narran en sus historias (Arroyo, 2008).

$Y$ aun cuando la diversidad cultural en los sistemas educacionales en todo el mundo ha adquirido legitimidad en los últimos años, es indiscutible al mismo tiempo que la operacionalización e implementación de políticas públicas inclusivas en el ámbito escolar es aún insuficiente (Akkari y Santiago, 2010; Santiago y Canen, 2013), especialmente porque los discursos de las autoridades solo quedan en exhortaciones 0 declaraciones de buenas intenciones y no se traducen en políticas y recursos que las posibiliten.

\section{SEGUNDA PARTE}

\section{Cuestionamientos a los proyectos integradores}

El despertar de la conciencia acerca de la necesidad de promover y defender los derechos humanos en todas sus formas, no limitando las eventuales violaciones a los derechos políticos o económico-sociales, como fue la tónica hasta el último cuarto del siglo $\mathrm{XX}$, además del intento de atender los efectos psico-emocionales que genera la 
exclusión y a la reflexión ética que deviene de ese proceso, han sido el marco conceptual en el que se han inscrito los esfuerzos de los organismos internacionales y de los gobiernos en procura de la inclusión educativa de todos aquellos que históricamente fueron excluidos.

No obstante, este esfuerzo no ha estado libre de controversias y resistencias: pensar críticamente la exclusión como un mecanismo de producción de la desigualdad social supone sumergirse en la complejidad y debates del mundo actual, sin eludir en ese pensar crítico la reflexión ética que, entre otras cosas, exige una discusión acabada en torno a los valores y efectos del orden capitalista en la vida de las personas. También implica aportar una palabra cuestionadora acerca de los otros paradigmas que legitiman o naturalizan la exclusión, como son, por ejemplo, el orden patriarcal-machista, el heterosexismo, la xenofobia y el racismo, entre muchos otros.

En el ámbito estrictamente educativo la mirada ha de ser puesta en las implicancias de los modelos de integración, en tanto apuestas normalizadoras, que si bien aspiran al reconocimiento de estudiantes o grupos tradicionalmente excluidos, ampliando las posibilidades de integración a otras dimensiones además de las socioeconómicas, no alteran en nada los paradigmas legitimadores de la desigualdad: estos continúan consagrando una suerte de jerarquización de los derechos, valores y expectativas de los distintos grupos sociales, por lo que, recurriendo a una categoría psicoanalítica, se suele denominar a este tipo de políticas como "inclusión perversa" (Sawaia, 2002), en el sentido de que mantiene inalteradas las lógicas de la desigualdad. No menos relevante en los análisis que cuestionan los procesos psicosociales de la exclusión son las reflexiones acerca de los mecanismos que llevan a una sociedad que dice valorizar los principios democráticos a convivir con las injusticias y discriminaciones.

\section{La irrupción de los otros excluidos}

Sin duda, uno de los aspectos positivos de los esfuerzos por incluir a los niños y jóvenes con NEE ha sido la mayor sensibilidad respecto de los efectos nocivos de la exclusión, tanto para las víctimas cuyos derechos son vulnerados, como para aquellas sociedades que se acostumbran a convivir con la injusticia llegando inclusive a naturalizarla. Ha sido este despertar de conciencias el que ha permitido visibilizar a otros excluidos, que, aunque presentes en los sistemas escolares formales, su existencia ha sido negada, invisibilizada 0 simplemente se ha intentado homogeneizarlos a través de mecanismos que desconocen sus peculiaridades 0 negado sus identidades como una forma de anulación y desprecio (Silva, 2000), ya sea hacia sus culturas, sus lenguas, sus cosmovisiones, sus valoraciones, sus percepciones de la realidad, o hacia sus inclinaciones erótico-afectivas o sus deseos. A este respecto cabe recordar, como sostiene Morgade y Alonso (2008), que la pedagogía que se construyó en la modernidad y que funciona todavía como 
orientadora de las prácticas docentes produjo la "pedagogización del cuerpo". Esta pedagogización toca no solo los estudiantes sino a los propios profesores, y sus efectos se dejan sentir tanto en la represión de la sexualidad como en la construcción de identidades.

Entre los nuevos excluidos (Bhabha, 2013) se pueden citar a los migrantes, las minorías étnicas, los disidentes sexuales o genéricos, etc. En el caso de los indígenas, como ha ocurrido reiteradamente en Chile, pese a que la escuela se ha mostrado preocupada por ellos, ha sido en vista de su asimilación a la cultura nacional. En el mejor de los casos, en las últimas décadas ha habido un progresivo interés por el rescate de su cultura, pero desde una perspectiva subordinada, donde su cultura aparece a los ojos de la comunidad nacional como lo anecdótico, peculiar o folclórico, en un diálogo asimétrico con la cultura dominante, pero nunca como un modelo a seguir o un proyecto educativo viable.

Esta situación pareciera reproducirse inclusive en el ámbito de la educación superior. Como sostienen Williamson y Coliñir (2015), las peculiaridades culturales de los universitarios mapuches no solo serían invisibilizadas, sino que los problemas socioeconómicos que aquejan particularmente a las comunidades indígenas guardarían estrecha relación con esa condición, impactando tanto en el acceso a la Universidad como en la mantención o término oportuno de las carreras de estos estudiantes. Es más, la propia producción de conocimiento estaría marcada por una impronta etnocentrista que invisibilizaría o desconocería los saberes de estos pueblos.

En lo que respecta a las personas con capacidades físicas diferentes, el reconocimiento ha estado presente desde hace ya algunas décadas, ya sea gracias a campañas solidarias de recaudación de fondos ${ }^{5} 0$ a través de escuelas segregadas. Buen ejemplo de ello son las escuelas especiales para sordos y no videntes. Con todo, estas escuelas continúan apostando por una lógica que perpetúa la jerarquización de las capacidades y la segregación. La posibilidad de una inclusión efectiva de estos niños y jóvenes a las escuelas comunes es aún distante dada las limitaciones espaciales, los requerimientos de infraestructura y las exigencias de materiales educativos especiales. Hablar en estos casos de inclusión educativa en consideración a las carencias y falta de voluntades resulta temerario.

En ese contexto no deja de ser llamativo que aún las universidades formadoras de profesores, que denuncian el descuido y apelan por un sistema escolar más inclusivo, sean en su mayoría incapaces de acoger en su seno a estos estudiantes, tanto porque su infraestructura, equipamiento y materiales educativos no está adaptado para las exigencias de una persona con capacidades diferentes, como a un currículo que no reconoce las diferencias.

No se puede obviar tampoco que la lógica que subyace a los proyectos integradores, entendiendo por tal los intentos asimilacionistas, homogeneizantes o

\footnotetext{
${ }^{5}$ Ejemplo emblemático de esta práctica ha sido por más de cuatro décadas la Teletón.
} 
normalizadores, comúnmente están basados en la idea de que son grupos minoritarios que deben ser asistidos, apoyados o integrados, desde una perspectiva paternalista, a un supuesto orden de normalidad, cuyo acento está dado por su condición de minoría o excepcionalidad.

Sorprende, en este sentido, que las mujeres, no siendo en ningún caso minorías en los sistemas educativos, sean tratadas como si lo fuesen. Todos los dispositivos de la escuela parecieran estar diseñados por y para los hombres, donde lo femenino y todo lo asociado a ello está silenciado o muchas veces presentado de un modo estereotipado. No resulta extraño, entonces, el trato desigual e inequitativo que reciben niños y niñas, aún en los procesos de enseñanza-aprendizaje, donde la atención y las expectativas están puestas en los varones, en desmedro o desconociendo las capacidades y potencialidades de las mujeres (Flores, 2007) ${ }^{6}$.

Un grupo con el cual las experiencias de integración son aún incipientes en el caso chileno, es el de los migrantes. Dado lo sostenido de las corrientes migratorias hacia Chile, especialmente de países vecinos en las últimas dos décadas (Solimano y Tokman, 2008; Stefoni, 2011), han obligado al sistema educativo nacional a buscar alternativas de integración, algunas de ellas recogidas de otras realidades con una vasta experiencia en el tema y otras improvisadas motivadas por las exigencias del momento. $Y$ aun cuando en un inicio los niños y jóvenes migrantes se tendieron a concentrar en escuelas muncipalizadas de algunas comunas del Gran Santiago o ciudades del norte, paulatinamente, y en consideración a la propia diversificación socioeconómica de los migrantes y a una redistribución regional se han comenzado a ser más habituales en otras zonas del país y en otro tipo de establecimientos.

Lo que cabe preguntarse en este caso es si las políticas educacionales privilegiarán un modelo homogeneizador-asimilacionista a la cultura nacional, como ha ocurrido históricamente con los indígenas, por ejemplo, o, por el contrario, se va a promover una inclusión educativa que incentive el diálogo simétrico entre culturas y nacionalidades, donde los niños y jóvenes migrantes cumplan la función de puente y mediación entre culturas.

Otro grupo no menos desafiante inscrito en las llamadas minorías étnicas es el de los gitanos. Si bien poco relevantes en términos numéricos, interpelan al sistema educacional en su conjunto, porque al mismo tiempo que reclaman su condición de nacionales, reivindican el derecho a la preservación de una cultura propia. Al igual que, como ocurre con los indígenas y los migrantes, los gitanos cuestionan al sistema no solo por la efectividad de las políticas y estrategias inclusivas, sino por la identidad nacional que se va tejiendo en las salas de clases.

Desafío no menor si se tiene en consideración que lo que está en juego con la inclusión de estos niños y jóvenes no es solo un proyecto educativo que se fundamenta

6 Sugerentes en este sentido son los estudios en torno a género y educación desarrollados por el Centro Interdisciplinario de Estudios de Género (CIEG) de la Universidad de Chile. 
en los derechos humanos, el reconocimiento de la diversidad en todos los planos y un estilo de enseñanza que se nutre de esa misma diversidad, sino la construcción de una identidad nacional que, aunque cimentada en la propia historia y cultura, se deja interpelar y enriquecer por los aportes de otras culturas. Una identidad abierta, reflexiva, autocrítica, que transita sin temores entre lo particular y lo universal. Que no se deja aprisionar por los localismos y particularismos, pero tampoco se encandila 0 diluye en un cosmopolitismo desenraizado. En otras palabras, una identidad inclusiva que aspira a formar ciudadanos para el mundo pero a partir de una matriz cultural propia (Samper, 2002; Cornejo, 2012b).

Uno de los grupos cuyo reconocimiento es muy reciente y no libre de resistencias y controversias, tradicionalmente silenciados, invisibilizados y punidos en razón de su disidencia del orden heteronormativo, es el de las lesbianas, gays, bisexuales, transgéneros, transexuales e intersexuales (LGBTI) (Lionço y Diniz, 2009). Sin duda, si hay grupo que pone en jaque la inclusión educativa son estos niños y jóvenes, no solo porque no existen políticas definidas de inclusión, salvo las exhortaciones a la tolerancia, sino porque no existe la convicción de que sus peculiaridades deban ser aceptadas y menos aún valoradas por el sistema educacional.

A diferencia de todos los otros excluidos que al menos gozan de una aceptación teórica, pues, en la práctica su inclusión dista mucho de ser real, los niños y jóvenes LGBTI son resistidos, silenciados $u$ hostigados aun por los propios agentes educativos, ya sea por la homofobia cultural e institucional que configura la escuela, o porque el educador es también portador y agente de esa misma homofobia en razón de su historia personal, sus valores y creencias (Morgade y Alonso, 2008; Núñez, 2011).

De este modo, no es casual que de todos los tipos de bullying el homofóbico haya sido definido como el más nocivo, al punto de haber sido caracterizado como un problema de salud pública (American Educational Research Association, 2013), ya que, en los otros, comúnmente la familia solidariza con la víctima y hace patente su malestar con el establecimiento. En el bullying homofóbico, por el contrario, la víctima calla no solo por el temor al acoso de los pares de que está siendo objeto, sino por el miedo a ser maltratado por su propia familia, produciéndose una doble victimización. De allí, que no sorprenda, de acuerdo al estudio de UNESCO (Organización de las Naciones Unidas para la Educación, la Ciencia y la Cultura, 2012), que América Latina sea el continente donde es más recurrente este tipo de violencia y Chile sea el país que encabece la lista.

En el ámbito educativo, probablemente al igual que como ocurre en otros campos, la homofobia es tan usual que hablar de inclusión educativa resulta ilusorio, tanto porque no existen políticas definidas, como por las resistencias fundadas en supuestos religiosos o conservadurismo político a que estos niños y jóvenes sean 
efectivamente incluidos ${ }^{7}$. En el mejor de los casos se los tolera en los establecimientos, dado que no pueden ser expulsados del sistema de acuerdo a las disposiciones ministeriales, produciéndose una suerte de "tolerancia de lo inevitable". Sin embargo, su presencia incomoda, al punto que no solo sus inclinaciones erótico-afectivas son negadas o punidas, sino también porque los dispositivos normalizadores continúan operando a través del silenciamiento, que no es más que otra forma de violencia (Dinis, 2011), o a través de una heterosexualidad obligatoria que no reconoce ni acepta la disidencia (Rich, 2010).

Con este grupo, al igual que como ocurre con todos los otros grupos, la queja permanente tanto de las víctimas de la exclusión como de los propios agentes educativos es la falta de preparación que, en general, evidencian los profesores en ejercicio como los estudiantes de pedagogías que están siendo preparados en las universidades para lidiar con la diversidad. Esa carencia no solo es en relación con los niños y jóvenes con NEE o con capacidades diferentes, sino con todos los grupos culturalmente distintos o con una orientación sexual o identidad genérica disidente. Para el caso chileno, algunos de los estudios que evidencian las falencias anteriores son Infante (2010), Granada, Pomes y Sanhueza (2013) y, más recientemente, el estudio de Educarchile (2016) que señala que el $86 \%$ de los profesores encuestados a nivel nacional necesita mayor preparación para enfrentar la educación del siglo XXI, con la diversidad de los estudiantes como uno de los mayores desafíos.

\section{TERCERA PARTE}

\section{Apuesta por un nuevo orden}

Hablar de inclusión educativa implica no solo poner atención a las distorsiones que se constatan en proyectos integradores, que aunque bien intencionados, subrayan la

\footnotetext{
7 Desde inicios de la presente década se vienen registrando una serie de denuncias de discriminación por orientación sexual e identidad de género en establecimientos educacionales a lo largo de todo el país, registradas tanto por el MINEDUC como por distintas organizaciones de derechos humanos u ONG vinculadas a la comunidad LGBTI. Desde 2009 a la fecha, el MINEDUC mantiene un catastro de denuncias de situaciones de discriminación acaecidas en escuelas nacionales, entre las cuales se consigna este tipo específico de discriminación. Entre las situaciones denunciadas destacan expulsiones sumarias de estudiantes LGBTI y medidas disciplinarias arbitrarias adoptadas por directivos escolares en contra de estos estudiantes. Las razones en que se fundan las medidas disciplinarias, de acuerdo a lo que se desprende del propio texto de las denuncias registradas por el MINEDUC, son presunción de "homosexualismo", expresiones afectivas entre estudiantes del mismo sexo, oposición o resistencias de estudiantes que han "salido del closet" a invisibilizar su orientación sexual en el espacio escolar, entre otras. No son inusuales tampoco denuncias de establecimientos, especialmente confesionales, que, en sus reglamentos de convivencia escolar, contienen expresiones abiertamente homofóbicas o prohíben el ingreso de estudiantes LGBTI, pese a las instrucciones expresas del MINEDUC en sentido contrario, a la Ley 20.609 Antidiscriminación (2012) y a la Ley 20.845 de Inclusión Escolar (2015). Entre los informes que dan cuenta, entre otros aspectos, de la homofobia escolar se puede citar el XV Informe Anual de Derechos Humanos: Diversidad Sexual y de Género en Chile (Movimiento de Liberación e Integración Homosexual, 2016), donde se consigna, por ejemplo, que el 70,3\% de los estudiantes LGBTI encuestados señala sentirse incómodo e inseguro en la escuela por su orientación sexual y el $41 \%$ por su expresión de género. Para mayor información respecto de este punto consultar J. Cornejo (2017). Disidencias sexuales en el sistema escolar chileno: represión en invisibilización.
} 
asimilación, la homogeneización o la normalización de las minorías, las excepcionalidades o las disidencias a un supuesto orden de normalidad, sino también poner atención a los sustratos ideológicos que subyacen en nuestros sistemas políticos, sociales, económicos y culturales que legitiman la subordinación, la jerarquización o exclusión social como una forma de relacionamiento entre los sujetos y grupos.

Luchar por un mundo sin exclusiones y consecuentemente por una educación efectivamente inclusiva supone, además, atender a los efectos que genera la exclusión, pero también prestar atención a los paradigmas que legitiman o posibilitan la exclusión. En este sentido la preocupación no solo ha de ser puesta en los paradigmas políticos o socioeconómicos generadores de desigualdades y exclusión, sino también en los otros paradigmas más sutiles y transversales, probablemente más invisibles a nuestras miradas y sensibilidades: la xenofobia, el racismo, el sexismo, la homofobia, etc.

Es decir, atendiendo a la idea de las generaciones de derechos humanos, no basta con una preocupación por los derechos de primera o segunda generación, se ha de promover también una mayor sensibilidad y preocupación por aquellos derechos descuidados, omitidos, invisibilizados o silenciados.

Por otra parte, no se puede obviar que muchas veces las exclusiones se superponen en razón de las múltiples identidades que tiene una persona, ya que un mismo sujeto puede ser al mismo tiempo marginado por su orientación sexual y sufrir discriminación por su origen étnico o condición socioeconómica. En este caso, los esfuerzos inclusivos han de atender a todas las exclusiones. No obstante, retomando la idea inicial, la inclusión educativa no puede focalizarse apenas en las consecuencias de la exclusión a modo de plan reactivo, pues no basta con la implementación de medidas remediales o sanciones frente a la discriminación, la marginación o la violencia que se genera a partir de esas situaciones. Tampoco es suficiente apelar a una actitud proactiva en el sentido de anticiparse a las eventuales situaciones de discriminación o exclusión.

Todo esto aun cuando necesario y recomendable no rompe con los círculos de la exclusión, sino apenas atiende a sus efectos. Dicho en otros términos, no hay auténtica inclusión educativa si el propio sistema educativo no internaliza, promueve e implementa políticas, culturas y prácticas (Booth y Ainscow, 2002; Santos y Paulino, 2008) que quiebren los paradigmas que generan la exclusión.

En este sentido se puede afirmar que la inclusión educativa no es posible en un medio que legitima la desigualdad o la segregación aun fundada en supuestos religiosos o filosóficos que pretenden justificar, por ejemplo, la condena y consecuentemente la exclusión de los disidentes sexuales o genéricos, como tampoco es posible incluir en un sistema educativo como el chileno donde apelando a la libertad de enseñanza se pretende perpetuar un sistema que segrega en términos económicos, o donde la calidad de la educación no es un bien universal sino el privilegio de unos pocos (Programa de las Naciones Unidas para el Desarrollo, 2017). 
De acuerdo al Estudio Económico de la Organización para la Cooperación y el Desarrollo Económicos (OCDE, 2015), algunos de los problemas que evidencia Chile en el ámbito educativo muestran relación no solo con puntajes en la prueba PISA muy inferiores al del promedio de la OCDE, sino también con fuertes diferencias entre los resultados de estudiantes de estratos socioeconómicos más altos y más bajos. Este aspecto se suma a la necesidad de escuelas más inclusivas y mejoras para la profesión docente.

Hablar de inclusión en estos contextos, más allá exhortaciones o declaraciones de buena voluntad, resulta ofensivo y una provocación para aquellos que viven a diario la exclusión. Una verdadera inclusión educativa es aquella que aspira no apenas a proyectos focalizados de integración, sino a transformar desde sus fundamentos la cultura escolar, promoviendo una cultura inclusiva donde todos se reconocen. Para tornar ello posible es necesario replantearse, inclusive, los fundamentos de la propia educación, puesto que es allí muchas veces donde se alojan los paradigmas que legitiman y perpetúan la exclusión.

A partir de esa reformulación se han de implementar las innovaciones curriculares y las prácticas pedagógicas, no limitándolas solo a un cambio de contenidos o propuestas de nuevas estrategias de enseñanza-aprendizaje o de convivencia escolar, sino a crear una cultura donde todos son respetados y valorados, donde la diversidad es un valor y la inclusión no es el fin sino el medio que hace posible la experiencia de una cultura de la paz.

Papel no menor les cabe en este proceso a los profesores e instituciones formadoras de profesores. Los primeros, a la vez que agentes del cambio, han de ser modelos de inclusión, es decir, sujetos que están permanentemente evaluando sus prácticas, discursos y actitudes, a fin de identificar y erradicar todo sesgo excluyente o discriminador producto de su propia historia personal o formación.

El problema no está en reconocer en un uno mismo trazos internalizados de una cultura excluyente, sino en negar su existencia y no hacer nada por cambiarla. En este sentido, el profesor inclusivo es aquel que en su quehacer profesional y en su vida cotidiana vivencia sus convicciones acerca de un mundo sin exclusiones, no a modo de expectativa futura sino de utopía realizada, pues, resultaría incomprensible aspirar e intentar alcanzar ese mundo recurriendo a métodos autoritarios, arbitrarios o poco participativos. La democracia, la participación, el respeto irrestricto de los derechos humanos, la valoración de la diversidad, la inclusión, no pueden ser considerados fines a alcanzar sino los instrumentos mediante los cuales se construye ese mundo sin exclusiones.

En lo que toca a las instituciones formadoras de profesores, ellas han de ser espacio de crítica y reflexión acerca de los diversos modos que asume la exclusión social y de denuncia de todo intento de "inclusión perversa" (Sawaia, 2002), pero al mismo tiempo espacios donde la inclusión esté plasmada en sus políticas, en el currículo de formación de sus profesionales, en sus prácticas pedagógicas y en las 
interrelaciones entre estamentos y personas, pues no se entiende una institución que habla de inclusión, que forma profesionales para la inclusión, pero donde ella no hace parte de su cotidianeidad.

La inclusión educativa, más que un esfuerzo de teorización, es una exigencia ética de reconocimiento y realización de la propia condición humana. $Y$ si bien el proyecto puede parecer ambicioso en vista de los múltiples impedimentos, de una cultura que se resiste a los cambios y de paradigmas que legitiman la exclusión, no se puede olvidar que uno de los principios básicos de la educación, a diferencia de otras formas de transmisión de conocimiento, es que ella no se conforma con la transmisión o la reproducción de saberes: ella aspira a la transformación, a la innovación, a la creación de nuevo conocimiento. Desde esta perspectiva, la inclusión educativa no es simplemente una moda o el instructivo de lo políticamente correcto, ella es sobre todo una exigencia ética de respeto de la diversidad y valoración de la condición humana.

\section{REFERENCIAS}

Aguado, T. (2009). El enfoque intercultural en busca de buenas prácticas escolares. Revista Latinoamericana de Inclusión Educativa, 5(2), 23-42.

Ainscow, M., Dyson, A., Goldrick, S., y West, M. (2012). Developing equitable education systems. Londres, Inglaterra: Routledge.

Akkari, A., y Santiago, M. (2010). A gestão da diversidade cultural no contexto educacional brasileiro. Revista Educação em Questão, 38(24), 9-33.

American Educational Research Association. (2013). Prevention of bullying in school, colleges and universities. Washington, DC: American Educational Research Association.

Aránzazu, R., y Aguado, T. (2002). Pedagogía de la diversidad. Madrid, España: UNED.

Arroyo, M. (2008). Os coletivos diversos repolitizam a educação. En J. Diniz, y L. Geraldo (Orgs.), Quando a diversidade interroga a formação docente (pp.11-36). Belo Horizonte, Brasil: Autêntica.

Artiles, A., Kozleski, E., Dorn, S., y Christensen, C. (2006). Learning in inclusive education research: Remediating theory and methods with a transformative agenda. Review of Research in Education, 30, 65-108.

Artiles, A., y Kozleski, E. (2014). Educación inclusiva en el siglo XXI: notas para un programa de investigación histórico - cultural. En M. C. Cardona, y E. Chiner (Eds.), Investigación educativa en escenarios diversos, plurales y globales (pp. 51-65). Alicante, España: AIDIPE - Universidad de Alicante.

Bhabha, H. K. (2013). Nuevas minorías. Nuevos derechos. Buenos Aires, Argentina: Siglo XXI.

Biblioteca del Congreso Nacional de Chile. (2011). Ley 20.536 Sobre violencia escolar. Recuperado de: http://bcn.cl/1uvxm

Biblioteca del Congreso Nacional de Chile. (2012). Ley 20.609 De no discriminación. Recuperado de: http://bcn.cl/1uyqt 
Biblioteca del Congreso Nacional de Chile. (2015). Ley 20.845 de Inclusión Escolar. Recuperado de: http://bcn.cl/1uv1u

Blanco, R. (1992). Alumnos con necesidades educativas especiales y adaptaciones curriculares. Madrid, España: MEC.

Blanco, R. (2011). Educación inclusiva en América Latina y el Caribe. CEE Participación educativa, 18, 46 - 59.

Booth, T., y Ainscow, M. (2002). Index for Inclusión - developing learning and participation in schools. Bristol, Inglaterra: CSIE.

Borrillo, D. (2001). Homofobia. Barcelona, España: Ediciones Ballaterra.

Borrillo, D. (2009). A homofobia. En T. Lionço, D. Diniz (Orgs.), Homofobia \& Educação: um desafío ao silêncio (pp. 15-72). Brasilia, Brasil: Editora UnB.

Casanova, M. A. (2011). Educación inclusiva: un modelo de futuro. Madrid, España: Wolters Kluwer España.

Cornejo, J. (2012a). Educación, interculturalidad y ciudadanía. Educar em Revista, 43, 239-254.

Cornejo, J. (2012b). Retos impuestos por la globalización a los sistemas educativos latinoamericanos.

Revista Mexicana de Investigaciones Educativas, 17(52), 15-37.

Cornejo, J. (2017). Disidencias sexuales en el sistema escolar chileno: represión e invisibilización. Educação e Pesquisa, 43(3), 879-898. https://doi.org/10.1590/s1517-9702201707166973

Devalle de Rendo, A., y Vega, V. (2009). Una escuela en y para la diversidad. Buenos Aires, Argentina: AIQUE Educación.

Dinis, N. F. (2011). Homofobia e educação: quando a omissão também é signo de violencia. Educar em Revista, 3, 39-50.

Echieta, G. (2013). Inclusión y exclusión educativa. De nuevo voz y quebranto. Revista Iberoamericana sobre Calidad, Eficacia y Cambio en Educación - RINACE, 11(2), 99-118.

Educarchile. (2016). 86\% de los docentes afirma que necesita mayor preparación para enfrentar la educación del siglo XXI. Recuperado de http://www.educarchile.cl/ech/pro/app/detalle?id=228704

Flores, R. (2007). Representaciones de género de profesores y profesoras de matemática, y su incidencia en los resultados académicos de alumnos y alumnas. Revista Iberoamericana de Educación, 43, 103-118. Recuperado de https://rieoei.org/RIE/article/view/753

García Huidobro, J. E. (2007). Desigualdad educativa y segmentación del sistema escolar. Consideraciones a partir del caso chileno. Revista Pensamiento Educativo, 40(1), 65-87.

Gazzola, A. L., y Didriksson, A. (Eds.). (2008). Tendencia de la educación superior en América Latina y el Caribe. Recuperado de http://flacso.redelivre.org.br/files/2012/08/753.pdf

Granada, M., Pomes, M., y Sanhueza, S. (2013). Actitud de los profesores hacia la inclusión educativa. Papeles de Trabajo, 25, 51-59.

Infante, M. (2010). Desafíos a la formación docente: inclusión educativa. Estudios Pedagógicos, 36(1), 287-297. https://doi.org/10.4067/S0718-07052010000100016 
Hopenhayn, M. (2002). El reto de las identidades y la multiculturalidad. Pensar Iberoamérica. Revista de Cultura, 0. Recuperado de http://www.oei.es/historico/pensariberoamerica/ric00a01.htm

Honneth, A. (2010). Reconocimiento y menosprecio. Sobre la fundamentación normativa de una teoría social. Madrid, España: Katz Editores.

Irigoyen, J., Jiménez, M., y Acuña, K. (2011). Competencias y educación superior. Revista Mexicana de Investigación Educativa, 16(48), 243-266.

Lavilla, L. (2013). La integración escolar. Revista de Claseshistoria. Recuperado de http://www.claseshistoria.com/revista/2013/articulos/lavilla-integracion-escolar.html

Lionço, T., y Diniz, D. (Orgs.). (2009). Homofobia e educação: um desafío ao silêncio. Brasilia, Brasil: Editora UnB.

Maldonado, N. (2007). Sobre la colonialidad del ser: contribuciones al desarrollo de un concepto. En S. Castro-Gómez, y R. Grosfoguel (Orgs.), El giro decolonial: reflexiones para una diversidad epistémica, más allá del capitalismo global (pp. 127-168). Bogotá, Colombia: Siglo del Hombre Editores-Universidad Javeriana.

Ministerio de Educación de Chile. (2016). Orientaciones para la construcción de comunidades educativas inclusivas. Recuperado de https://www.mineduc.cl/2017/03/07/orientaciones-comunidadeseducativas-inclusivas

Morgade, G., y Alonso, G. (Comps.). (2008). Cuerpos y sexualidades en la escuela: De la normalidad a la disidencia. Buenos Aires, Argentina: Paidós.

Movimiento de Liberación e Integración Homosexual. (2016). XV Informe Anual de Derechos Humanos: Diversidad Sexual y de Género en Chile. Recuperado de http://www.movilh.cl/wpcontent/uploads/2017/03/XV-Informe-de-DDHH-2016-MOVILH.pdf

Núñez, G. (2011). ¿Qué es la diversidad sexual? Reflexiones desde la academia y el movimiento ciudadano. Quito, Ecuador: Ediciones Abya - Yala.

Organización de las Naciones Unidas para la Educación, la Ciencia y la Cultura. (1990). Conferencia Mundial sobre Educación para Todos: Satisfacción de las necesidades básicas de aprendizaje. Recuperado de http://www.unesco.org/education/pdf/JOMTIE_S.PDF

Organización de las Naciones Unidas para la Educación, la Ciencia y la Cultura. (1994). Declaración de Salamanca y marco de acción para las necesidades educativas especiales. Recuperado de http://www.unesco.org/education/pdf/SALAMA_S.PDF

Organización de las Naciones Unidas para la Educación, la Ciencia y la Cultura. (2008). La educación inclusiva: el camino hacia el futuro. Recuperado de http://www.ibe.unesco.org/fileadmin/user_upload/Policy_Dialogue/48th_ICE/CONFINTED_48_Inf _2__Spanish.pdf

Organización de las Naciones Unidas para la Educación, la Ciencia y la Cultura. (2009). Directrices sobre políticas de inclusión en la educación. Recuperado de http://unesdoc.unesco.org/images/0017/001778/177849s.pdf 
Organización de las Naciones Unidas para la Educación, la Ciencia y la Cultura. (2011). Sistema educativo de Chile fomenta la desigualdad. Recuperado de http://portal.unesco.org/geography/es/ev.phpURL_ID=15019\&URL_DO=DO_TOPIC\&URL_SECT ION=201.html

Organización de las Naciones Unidas para la Educación, la Ciencia y la Cultura. (2012). Respuestas del sector educación frente al bullying homofóbico. Recuperado de http://unesdoc.unesco.org/images/0022/002229/222918S.pdf

Organización de las Naciones Unidas para la Educación, la Ciencia y la Cultura. (2015). Declaración de Icheon - Educación 2030. Recuperado de http://unesdoc.unesco.org/images/0024/002456/245656s.pdf

Organización para la Cooperación y el Desarrollo Económicos. (2015). Estudios Económicos de la OCDE - Chile. Recuperado de https://www.oecd.org/eco/surveys/Chile-2015-vision-general.pdf

Orozco, B. (2000). De lo profesional a la formación en competencias: giros conceptuales en la noción de formación universitaria. En M. A. Valle, Formación en competencias y certificación profesional (pp. 105-139). México D. F., México: Universidad Nacional Autónoma de México.

Pedró, F. (2012). Políticas públicas sobre apoyo y refuerzo educativo: evidencias internacionales. Revista de Educación, Número extraordinario, 22-45.

Poblete, R. (2009). Educación intercultural en la escuela de hoy: reformas y desafíos para su implementación. Revista Latinoamericana de Inclusión Educativa 3(2), 181-200.

Programa de las Naciones Unidas para el Desarrollo. (2017). Desigualdades: orígenes, cambios y desafíos de la brecha social en Chile. Santiago de Chile, Chile: Programa de las Naciones Unidas para el Desarrollo.

Puig, J. M. (Coord.). (2012). Cultura moral y educación. Barcelona, España: Graó.

Santos, M. P. (2013). Dialogando sobre a inclusão em educação: contando casos (e descasos). Curitiba, Brasil: Editora CRV.

Santos, M. P., y Paulino, M. M. (2008). Inclusão em educação: culturas, políticas e prácticas. São Paulo, Brasil: Cortez Editora.

Rich, A. (2010). Desfazendo o "natural": a heterossexualidade compulsória e o continuum lesbiano. Revista Bagoas, 5, 45-55.

Rhoads, R. (1994). Coming Out in College. The Struggle for a Queer Identity. Westport, CT: Bergin \& Garvey.

Samper, E. (2002). Educación y globalización. En E. Samper, y A. Camacho (Coords.), Educación y globalización: los desafíos para América Latina Vol. 1 (pp. 43-49). Santiago de Chile, Chile: Naciones Unidas-CEPAL-ECLAC-OEI.

Santiago, M., y Canen, A. (2013). Les aproches interculturelles dans I'education au Brésil. Revue International d'Education de Sèvre, 63, 43-54.

Sawaia, B. (Org.). (2002). As artimanhas da exclusão - analise psicosocial e ética da desigualdade social. Petrópolis, Brasil: Vozes. 
Silva, T. T. (2000). Identidade e diferencia: A perspectiva dos estudos culturais. Petrópolis, Brasil: Vozes.

Solimano, A., y Tokman, V. (2008). Migraciones internacionales en un contexto de crecimiento económico: el caso de Chile. En A. Solimano (Coord.), Migraciones internacionales en América Latina: booms, crisis y desarrollo (pp. 185-243). México D. F., México: Fondo de Cultura Económica.

Stefoni, C. (2011). Perfil migratorio de Chile. Buenos Aires, Argentina: OIM Oficina Regional para América del Sur.

Van Steenlandt, D. (1991). La integración de niños discapacitados a la educación común. Santiago de Chile, Chile: Organización de las Naciones Unidas para la Educación, la Ciencia y la Cultura. Recuperado de http://unesdoc.unesco.org/images/0008/000884/088454so.pdf

Williamson, G., y Coliñir, M. (2015). Interculturalidade no ensino superior: ações e reflexões desde os direitos humanos. Conjectura: Filosofía e Educação, 20(número especial), 101-130. 